Amit Agarwal, Gregor Lämmel, Kai Nagel

\title{
Incorporating within link dynamics in an agent-based computationally faster and scalable queue model
}

Journal article | Accepted manuscript (Postprint)

This version is available at https://doi.org/10.14279/depositonce-8217

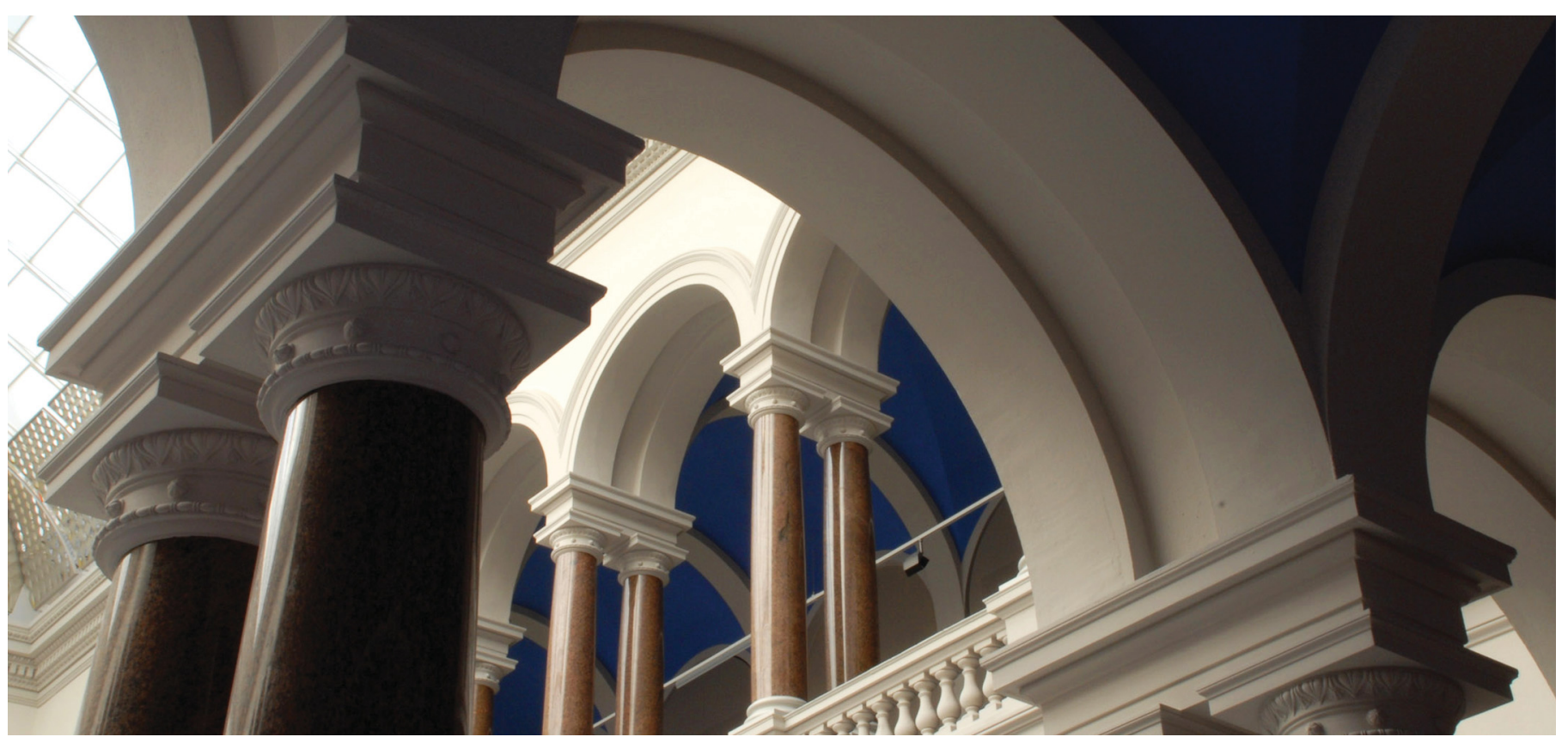

This is an Accepted Manuscript of an article published by Taylor \& Francis in Transportmetrica A on 21 Aug 2017, available online: http://www.tandfonline.com/10.1080/23249935.2017.1364802.

Agarwal, A.; Lämmel, G.; Nagel, K. (2017). Incorporating within link dynamics in an agent-based computationally faster and scalable queue model. Transportmetrica A: Transport Science, 14(5-6), 520541. https://doi.org/10.1080/23249935.2017.1364802 
To appear in Transportmetrica A: Transport Science

Vol. 00, No. 00, Month 20XX, 1-22

\title{
Incorporating within link dynamics in an agent-based computationally faster and scalable queue model
}

\author{
Amit Agarwal $^{\mathrm{a} *}$, Gregor Lämmel ${ }^{\mathrm{b}}$ and Kai Nagel ${ }^{\mathrm{a}}$ \\ a Technische Universität Berlin, Sek. SG 12, Salzufer 17-19, 10587 Berlin, Germany; \\ ${ }^{\mathrm{b}}$ German Aerospace Center (DLR), Institute of Transport Systems, Rutherfordstr. 2, 12489 \\ Berlin, Germany \\ (v2.0 released February, 2017)
}

\begin{abstract}
The growing pace of urbanization increases the need of simulation models to handle large scale scenarios in reasonable time. The present study proposes a fast spatial queue model, which is anchored to an agent-based travel demand simulation framework. The existing queue model is extended to produce more realistic flow dynamics by introducing backward traveling holes to mixed traffic conditions. In this approach, the space freed by a leading vehicle is not immediately available to the following vehicle. The resulting dynamics resembles the Newell's simplified kinematic wave model. The space freed corresponding to each leaving vehicle is named as 'hole' and, as following vehicles occupy the space freed by leading vehicles, the hole travels backward. This results in triangular fundamental diagrams for traffic flow.

The robustness of the model is tested with flow density and average bike passing rate contours. Spatio-temporal trajectories are presented to differentiate the queuing patterns. Finally, a comparison of the computational performance of the different link and traffic dynamics of the queue model is made.
\end{abstract}

Keywords: queue model; heterogeneous traffic; backward traveling holes; computational efficiency; kinematic waves; large-scale traffic simulation;

\section{Introduction}

The number of mega cities with a population of 10 million or more will increase from 10 in 1990 to 41 in 2030 (United Nations 2014). The rapid urbanization is driven by rapid growth in urban population and goes along with rapid infrastructure development. This has led to rapid increase in number of motor vehicles as well as passenger and freight traffic volumes. In order to manage such large amount of traffic, to predict future trends, and to test various policy measures, travel demand simulation models are required.

A variety of models exists, which can be differentiated based on model abstraction, reliability of the results, etc. Many such models use iterative algorithms to determine a dynamic user equilibrium. However, the simulation of a whole day of traffic for a large urban network takes a lot of CPU time (Gawron 1998). In particular, models with a high level of detail are resource intensive and require high performance computing systems. Access to such systems is not very common. Thus, a model which can simulate big urban agglomerations with less computational resources in reasonable time is required. This paper presents a realistic, computationally fast, agent-based traffic flow model, which can be used in homogeneous as well as heterogeneous traffic conditions. It considers 
every person individually, and updates the routes of several thousand agents dynamically, while maintaining its computational efficiency. The efficiency is in part a result of a computationally efficient queue model, which only tracks agents during link enter/leave events and never in between 1

A simple queue model is composed of a) service rate (supply-side) and b) request rate (demand-side); a queue appears if the request rate is higher than the service rate. In the traffic simulation using queue models, a vehicle travels on a link (road, edge) with restricted speed until it reaches the downstream end of the link. If inflow to the link is higher than maximum outflow (link capacity), a queue appears. It is a simple yet very efficient approach in traffic flow models due to its computational performance (Gawron 1998; Simon, Esser, and Nagel 1999).

Besides the already fast base performance of queue models, additional computing time savings can be obtained from queue models by scaling down the number of vehicles while at the same time scaling down each link's storage and flow capacity accordingly.

Various queue models (point queue, spatial queue etc.) exists which can be characterized based on several assumptions. A simple queue model is the point queue (PQ) model, in which vehicles are assumed to be stacked on top of each other. Thus the PQ model is also known as vertical queue model (Hurdle and Son 2001; Zhang and Nie 2005; Zhou and Taylor 2014). In the PQ model, it is assumed that an infinite number of vehicles can be stored on a link, thus the length of the queue is zero, and spillover does not occur. In consequence, inter-link and intra-link interactions are absent (Zhang and Nie 2005).

Use of the PQ model is limited because spillover is common in urban networks. This shortcoming is overcome in the spatial-queue (SQ) model (see, e.g., Simon, Esser, and Nagel 1999). Since the physical length of the queue is now non-zero, this is also known as horizontal queue (Kim, Lim, and Lim 2003; Maerivoet and Moor 2005). In these models, every link is equipped with a storage capacity which depends on the length of the link, number of lanes, etc. The SQ model verifies the available storage capacity of the downstream link before allowing any vehicle to enter. Thus, if downstream link is full, spillover occurs onto the upstream link.

In many SQ models, it is assumed that space originating from the leaving vehicle is available immediately at the upstream end of the link. This is unrealistic; it means that the intra-link interaction is still absent. Flow dynamics with intra-link interaction is described by the kinematic wave model (KWM; Lighthill and Whitham 1955; Richards 1956). The KWM realistically describes queue propagation. Newell (1993) describes a simplified kinematic wave model. To numerically solve the full kinematic wave equations, the cell transmission model (CTM) is proposed by Daganzo $(1994,1995)$ with a computational complexity proportional to the spatial discretization of the links. A link transmission model (LTM) is proposed by Yperman (2007); this model does not have a spatial discretization in order to reduce the computational burden. The traffic propagation under this model is consistent with KWM. Similarly, Balijepalli, Ngoduy, and Watling (2014) propose a two-regime transmission model (TTM) by considering two regimes of traffic flow (free flow and congested) and dynamically model the queue-length. A similar mesoscopic model is proposed by Tordeux et al. (2014) in which, a particle (vehicle) jumps on a set of sites (road sections) and the jump rate depends on the number of vehicles on the departure and arrival sections. It is shown that the model can reproduce

\footnotetext{
${ }^{1}$ Other important elements that make the model efficient/fast are:

- Links without activity are switched off (this could be done with any link dynamics, not just queue)

- Queue model allows to run samples.

- The model can be distributed onto multiple threads.
} 
several observed properties of the traffic flow. A stochastic CTM model is proposed by Sumalee et al. (2011) to model the stochastic demand and supply. The model is then tested on a hypothetical freeway corridor and an empirical study scenario.

The differences between the PQ, SQ and other models are summarized in many studies (e.g. Zhang and Nie 2005; Zhang, Nie, and Qian 2013; Frederix, Viti, and Tampère 2010). The former two compare the PQ, SQ and CTM under dynamic network loading conditions. It is shown that the PQ model considerably underestimate the dynamic network travel time and in heavily congested network with spillover; also the SQ model without kinematic waves can underestimate the impact of congestion. The latter compares the PQ, SQ and LTM and shows that both PQ and SQ models tend to misidentify the flow i.e. lower if congestion is emerging and higher if congestion is dissolving. The PQ and CTM models are also compared by Nie and Zhang (2005). The authors show that the PQ model takes the least amount of the CPU time and memory whereas the CTM model takes most amount of the CPU time and memory.

The limitation of missing intra-link interaction in the SQ model can be surmounted by integrating backward travelling holes (Charypar, Axhausen, and Nagel 2007; Eissfeldt et al. 2006) in the SQ model. Thus, such SQ models are more realistic and show KWM like flow dynamics. In fact, Flötteröd (2016) shows equivalence with the double ended queue model. The present study integrates this concept into an existing SQ model at the cost of very little additional computational effort. A multi agent-based travel demand simulator, MATSim ${ }^{2}$ is used. It uses a spatial queue model (Gawron 1998; Simon, Esser, and Nagel 1999: Cetin, Burri, and Nagel 2003) with currently two different link dynamics: first-in-first-out (FIFO), and passing (Agarwal et al. 2015).

The presence of heterogeneity of vehicles on the urban road network of most of the developing economies restricts the use of homogeneous traffic flow models. Only a few models are developed to deal with such traffic conditions. In this direction, the LWR model is extended analytically for mixed traffic by Zhang and Jin (2002). Similarly, an analytic dynamic network loading model is proposed by Bliemer (2007) which consists of a link and a node model to incorporate multiple vehicle types. However, it lacks in showing the kinematic waves. A link transmission model for multiple user classes is proposed by Smits, Bliemer, and van Arem (2011). A detailed, heterogeneous simulation model is developed for mid block sections and found to be satisfactory in terms of replicating the field conditions (Venkatesan, Gowri, and Sivanandan 2008). However, to reduce the computational complexities, the present study focuses on extending the proposed queue model with holes for mixed traffic rather than addressing the more general LWR models.

Following the research direction by Charypar, Axhausen, and Nagel (2007), the present study extends and investigates the queue model with holes for mixed traffic conditions. The study focuses on 1) the sensitivity of the proposed model, 2) spatio-temporal plots for the proposed queue model and 3) comparison of the computational performance between different link and traffic dynamics of the queue model.

The remainder of the paper is structured as follows. Sec. 2 gives an overview of the travel demand simulation model for the present study and the original queue model anchored in it. Sec. 3 exhibits the experimental set up to generate various fundamental diagrams and density contours. Further, Sec. 4 explains the methodology for the queue model with holes and fundamental diagrams for it. The robustness of the proposed model is tested in Sec. 5. Sec.6 demonstrates the space-time trajectories for the proposed model. The comparison of the computational efficiency of various link and traffic dynamics for the queue model is done in Sec. 7. The study is concluded and way forward is laid in Sec. 8 .

\footnotetext{
2 'Multi-Agent Transport Simulation', see www.matsim.org
} 


\section{Travel demand simulator - MATSim}

The proposed queue model is anchored to a multi-agent travel demand simulation framework, MATSim (Horni, Nagel, and Axhausen 2016). Detailed information about the software has been published in many previous studies (e.g. Balmer et al. 2009; Balmer, Raney, and Nagel 2005, Raney and Nagel 2004, 2006). Road network and daily plans of the individual travelers are required as minimal inputs. The network loading algorithm is embedded into an iterative co-evolutionary algorithm (Balmer et al. 2009) (also see Fig. 1). In the present study, only the part related to the mobility simulation (mobsim) is discussed. It is a so-called queue model (Gawron 1998; Simon, Esser, and Nagel 1999 Cetin, Burri, and Nagel 2003). The default queue model in MATSim is a spatial queue (SQ) model without intra-link interaction and therefore, from here onwards, queue model refers to the spatial queue model ${ }^{3}$ A comparison between MATSim and other models (TRANSIMS, SUMO, VISSIM) is made and it is found that MATSim is about 20 times faster than closest neighbor, TRANSIMS (Maciejewski 2010).

Fig. 1 shows different traffic and link dynamics with queue model. In a previous study by Agarwal et al. (2015), the FIFO behavior of queue model without holes (see Sec. 2.1.1) is extended by passing queue without holes (see Sec. 2.1.2). Queue model without holes is presented briefly in Sec. 2.1. The seepage link dynamics in the queue model with holes is presented in Agarwal and Lämmel (2015, 2016).

\subsection{Queue model without holes}

In the MATSim simulation framework, the physics of a link 1 is determined by the free speed link travel time $\left(t_{l, f r e e}\right)$, flow capacity and storage capacity. These, in turn, are computed from the link length $\left(\ell_{1}\right)$, the number of lanes, and the link's maximum speed $\left(\mathrm{v}_{\mathrm{l}, \max }\right)$. The flow capacity or link outflow is the maximum number of vehicles allowed to leave the link per time step, whereas the storage capacity of a link is the maximum number of vehicles that can be placed on the link. Different vehicle sizes are taken into account by vehicle-specific passenger car equivalents (PCEs), which are applied both to flow and to storage. Similarly, vehicle-specific maximum speeds may be lower than the link speed; always, the smaller of the two is used.

\subsubsection{FIFO}

For reference purpose, two link dynamics - namely, first-in-first-out (FIFO) and passing of the queue model without holes - are summarized here. The default variant of the queue model in MATSim processes the vehicle queue on each road segment (link) according to FIFO order. Free speed travel time $\left(t_{l, f r e e}\right)$ for each entering vehicle is computed as the ratio of the $\ell_{1}$ and $v_{1, \max }$. Subsequently, the vehicle is added to the queue data structure, and its storage consumption is noted. It can leave the link and enter the downstream link provided -

- the vehicle has spent at least $t_{1, \text { free }}$ on the link,

- the flow capacity of the link is observed and

- the downstream link has enough space for the vehicle.

Fig. 2 shows the flow density curve for different types (classes) of cars and bikes differentiated based on their speeds. For each vehicle class, a different simulation run is set up

\footnotetext{
${ }^{3}$ The queue model in MATSim can behave as point queue model by setting sufficiently high value of the link storage capacity. However, this is omitted in the present study due to its unrealistic nature.
} 


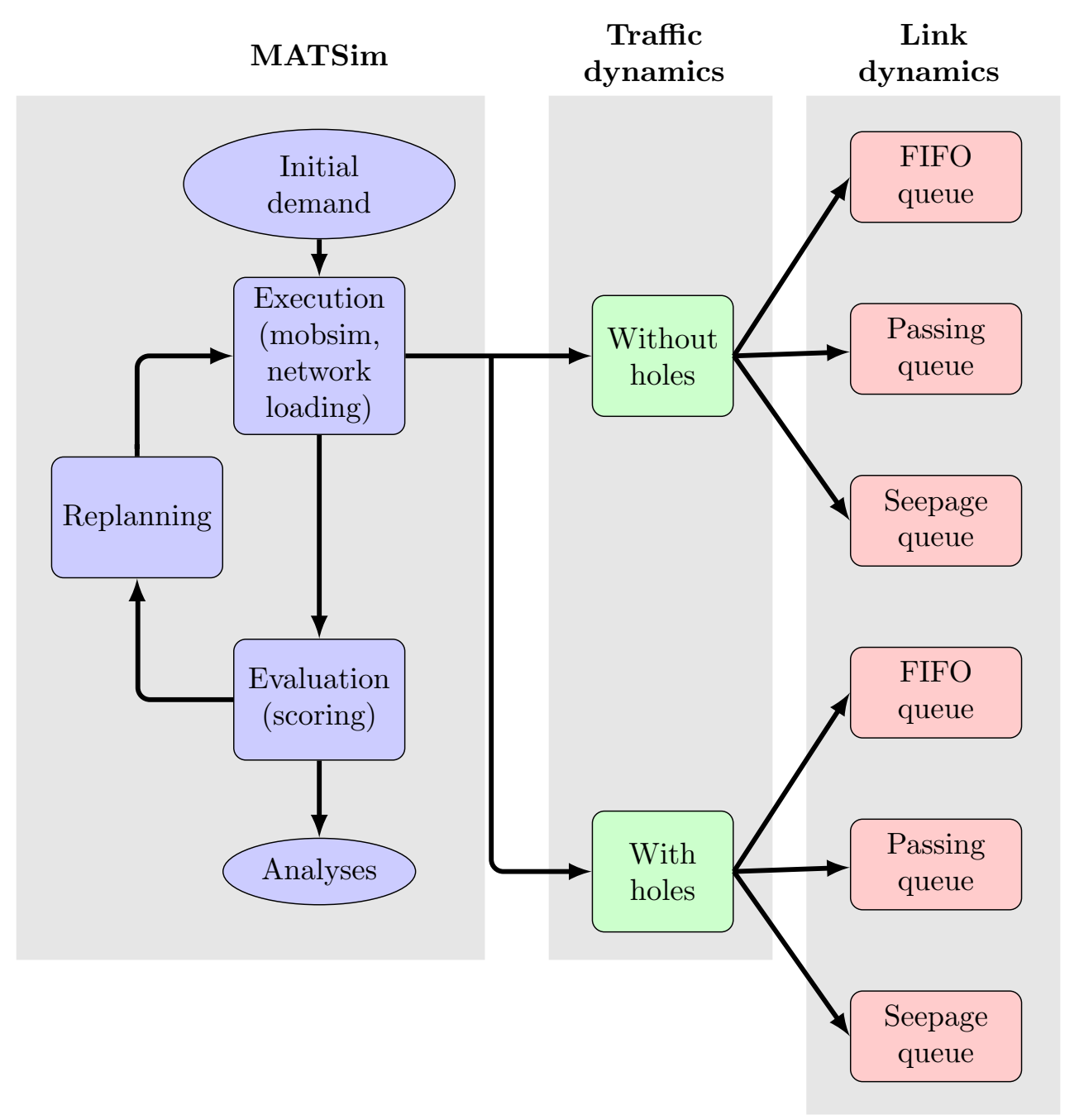

Figure 1. MATSim evolutionary algorithm and, various traffic and link dynamics.

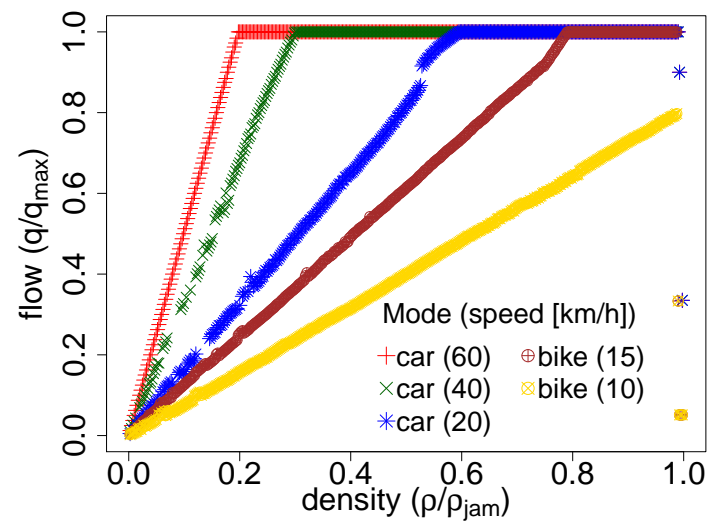

Figure 2. Flow density curves for car and bike. 
as explained in Sec. 3. The slope of the left branch of the FDs is approximately equal to the maximum speed of each vehicle class. Thus, this portion of the FD is called free flow regime and the flow density relationship is linear. The primary relationship between the three fundamental variables of traffic flow $(\mathrm{q}=\rho \cdot \mathrm{v})$ holds. Afterwards, this branch meets with a horizontal section, where flow remains constant (maximum flow) over a certain density range. This horizontal section is called capacity regime. Further, the horizontal section meets with nearly vertical branch of FD, which is known as jammed regime (Simon and Nagel 1999). This branch is very steep and unrealistic, which is a shortcoming of the standard queue model (Simon, Esser, and Nagel 1999).

Comparing the different vehicle classes, one can observe that as the maximum speed of the vehicle class decreases, the slope of the left branch of the FD also decreases and consequently, the horizontal section of the capacity regime shrinks. For the slowest vehicle class, i.e. bike with maximum speed as $10 \mathrm{~km} / \mathrm{h}$, the slope is so low such that the capacity regime does not exist, and in consequence, maximum flow is smaller than the link outflow (Agarwal et al. 2015).

\subsubsection{Passing}

For heterogeneous traffic conditions, inclusion of different vehicle classes is necessary, which has been done by Agarwal et al. (2015). A vehicle class minimally needs maximum speed $\mathrm{v}_{\mathrm{v}, \max }$ and passenger car unit (PCU). For each vehicle class, flow and storage capacities are consumed based on its PCU. The free speed travel time is given by

$$
\mathrm{t}_{\mathrm{l}, \text { free }}=\frac{\ell_{\mathrm{l}}}{\min \left(\mathrm{v}_{\mathrm{l}, \max }, \mathrm{v}_{\mathrm{v}, \max }\right)} .
$$

The Passing is facilitated by sorting the queue data structure based on the earliest link exit time, computed as $t_{\text {entry }}+t_{l, f r e e}$, where $t_{\text {entry }}$ is the time when a vehicle enters the link. This makes the faster vehicles to overtake the slower vehicles (see Agarwal et al. 2015, for details).

Clearly, the queue model controls the vehicles only at entries and exits, and never in between which makes it computationally efficient. Additionally, in both link dynamics, it is assumed that the space freed by the leaving vehicle is available immediately on the the upstream end of the link therefore, the intra-link interaction is absent from the default variant of the queue model.

\section{Experimental design}

For various link and traffic dynamics of the queue model, fundamental diagrams (FDs) are drawn to show the relationship between the three fundamental quantities of the traffic flow, i.e. flow (q), density $(\rho)$ and speed $(\mathrm{v})$. The experimental set up to generate the FDs for various link and traffic dynamics of the queue model is presented next.

\subsection{Set up}

An equilateral triangular race track network is selected so that agents (individual travelers) can continue rotating on the race track until they are stopped. The network is used in many previous studies to generate FDs (see for e.g. Agarwal et al. 2015; Agarwal and Lämmel 2016). Each link of the network is $1000 \mathrm{~m}$ long and restricted (allowed) speed on the link is $60 \mathrm{~km} / \mathrm{h}$. The flow (outflow) and storage capacities of each link are $1600 \mathrm{PCU} / \mathrm{h}$ and $133.33 \mathrm{PCU} / \mathrm{km}$ respectively. 
The passenger car units (PCUs) and maximum speeds for car, motorbike and bike are assumed as 1, 0.25, 0.25 and 60,60, $15 \mathrm{~km} / \mathrm{h}$ respectively (Agarwal et al. 2013). Moreover, in order to check the behavior of heavy vehicles, truck mode is also used. The maximum speed and PCU of the truck vehicle class are assumed as $30 \mathrm{~km} / \mathrm{h}$ and 3 respectively.

\subsection{Steady state}

A simulation is run for each discrete density point in the FD. The flow and speed are measured at the end of the track. For each density point, the number of agents are determined. For multiple vehicle classes, the modal split and the density determine the number of agents for each vehicle class. Thus, these agents are allowed to run on the triangular race track until the fluctuations in the flow and speed of each mode are damped. This situation is referred to as steady state. The average values of the flow and speed at each density point are recorded at the steady state condition. If, a steady state is not achieved during an experimental run, data is not recorded.

\section{Queue model with holes}

As stated earlier in Sec. 2.1, the intra-link interactions are absent from the queue model without holes. It means, the freed space from a leaving vehicle is available immediately at the upstream end of the link. This is unrealistic; in real-life it takes some time for the free space to arrive on the upstream end of the link (Charypar, Axhausen, and Nagel 2007; Eissfeldt et al. 2006). Therefore, the present study introduces backward traveling holes into the existing queue simulation. In this approach, the space freed by a leaving vehicle is called as 'hole' or 'gap'. As the name depicts, the holes travel backward i.e. opposite to the direction of the traffic flow.

\subsection{Hole}

Similar to the vehicle class, each hole is also assigned with the PCU and the free speed travel time $\left(\mathrm{t}_{1, \text { hole }}\right)$ on the link. The PCU of the holes is the same as the leaving vehicle and $t_{1, h o l e}$ is given by

$$
\mathrm{t}_{\text {l,hole }}=\frac{\ell_{\mathrm{l}}}{\mathrm{v}_{\text {hole }}}
$$

where $\mathrm{v}_{\text {hole }}$ is the speed of the hole. In the present study, a constant hole speed of $15 \mathrm{~km} / \mathrm{h}$ is assumed which is a commonly used value in the literature (see Ch. 8 in Treiber and Kesting 2013; Zhang and Jin 2002; Han, Piccoli, and Szeto 2015). This corresponds to the speed of the backward traveling kinematic wave in the KWM. It depends mainly on the reaction time of the driver. For, the effective length of $1 \mathrm{PCU}\left(\ell_{\mathrm{eff}}=7.5 \mathrm{~m}\right)$, the hole speed $15 \mathrm{~km} / \mathrm{h}$ is equivalent to a reaction time of $1.8 \mathrm{sec}$ (see Ch. 4 in Treiber and Kesting 2013). Consequently, the effective value of hole speed depends on the size of the vehicle, e.g., the hole speed of a truck which is 3 times longer than a car, will be 3 times higher than that of the car $(=3 \cdot 7.5 \mathrm{~m} / 1.8 \mathrm{~s})$ (see van Wageningen-Kessels et al. 2014, for a similar example). 


\subsection{Backward traveling holes}

Corresponding to the every leaving vehicle, a hole is generated at the downstream end of the link. This hole is then occupied by the following vehicle; thus the hole propagates one step backward. The hole continues until it reaches the upstream end of the link. Consequently, after a critical density, the agents can not enter the link as long as no hole is available at the upstream end of the link. In contrast to the queue model without holes in which the freed space is available immediately on the upstream end of the link, in this approach, the space freed on the downstream end of the link is available on the upstream end of the link after $t_{l, h o l e}$. Thus, this approach implicitly introduces an inflow link capacity restriction, in addition to the existing outflow link capacity.

\subsection{Queue model with and without holes}
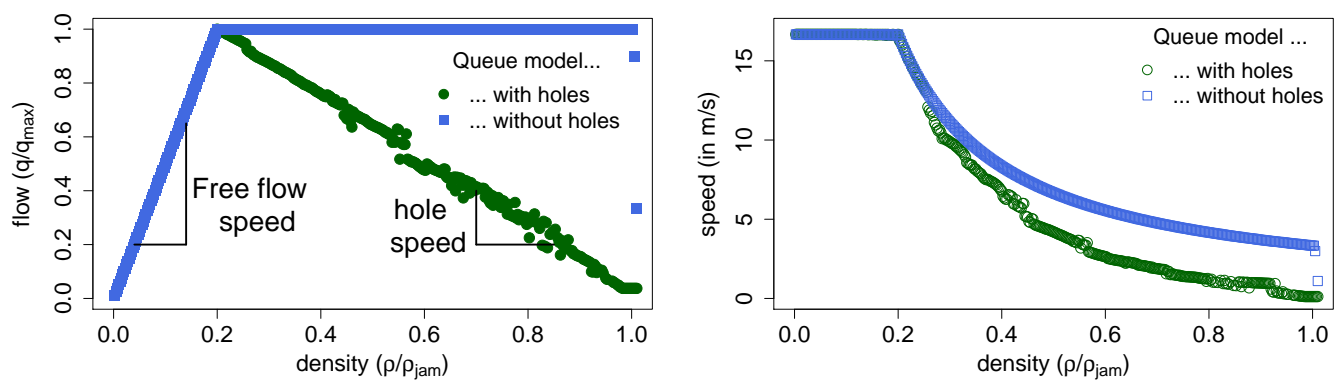

Figure 3. Comparison of FDs for only car simulation using queue model with and without holes.

Fig. 3 shows a comparison of both queue model approaches - with and without holesfor a car only simulation. Axes for flow and density are normalized. The FD for the queue model without hole is same as the FD for car with maximum speed $60 \mathrm{~km} / \mathrm{h}$ in Fig. 2. In the free flow regime, both traffic dynamics (with and without holes) show similar behavior i.e. the slope of the left branch of the FD is equal to the minimum of the two speeds $\left(\mathrm{v}_{\mathrm{v}, \max }\right.$ and $\left.\mathrm{v}_{\mathrm{l}, \max }\right)$. Now, since, the intra-link congestion is introduced by inserting the holes into the queue model, the free space on the upstream end of the link is not available immediately. Therefore, the slope of the congested branch is reduced to the speed of the backward traveling holes. It can also be observed that the critical density at which speed starts decreasing is same for both traffic dynamics. However, the rate of decrease in the speed for the queue model with holes is higher than in the queue model without holes. Clearly, the proposed model has produced the triangular fundamental diagram and a realistic jammed regime.

For queue model with holes, the flow (q) will be:

$$
\mathrm{q}= \begin{cases}\mathrm{v}_{\mathrm{l}, \mathrm{v}} \cdot \rho, & \text { if } \rho \leq \rho_{\mathrm{c}},(\text { free flow }) \\ \mathrm{v}_{\text {hole }} \cdot\left(\rho_{\mathrm{jam}}-\rho\right), & \text { if } \rho_{\mathrm{c}}<\rho \leq \rho_{\text {jam }}(\text { congested })\end{cases}
$$

where $\mathrm{v}_{\mathrm{l}, \mathrm{v}}=\min \left(\mathrm{v}_{\mathrm{l}, \max }, \mathrm{v}_{\mathrm{v}, \max }\right)$, i.e. minimum of link speed $\left(\mathrm{v}_{\mathrm{l}, \max }\right)$ and maximum speed of vehicle $\left(\mathrm{v}_{\mathrm{v}, \text { max }}\right), \rho_{\mathrm{c}}$ is critical density at which the flow is maximum $\left(\mathrm{q}_{\mathrm{max}}\right)$ and $\rho_{\mathrm{jam}}$ is the jam density. At critical density $\rho_{\mathrm{c}}$, the flow from free flow branch and congested branch will be equal (also see Eq. 8.11 and Eq. 8.16 Treiber and Kesting|2013), 
i.e.

$$
\begin{aligned}
\rho_{\mathrm{c}} \cdot \mathrm{v}_{\mathrm{l}, \mathrm{v}} & =\mathrm{v}_{\text {hole }} \cdot\left(\rho_{\mathrm{jam}}-\rho_{\mathrm{c}}\right) \\
\rho_{\mathrm{c}} & =\frac{\mathrm{v}_{\text {hole }} \cdot \rho_{\mathrm{jam}}}{\left(\mathrm{v}_{\text {hole }}+\mathrm{v}_{\mathrm{l}, \mathrm{v}}\right)}
\end{aligned}
$$

Therefore, the maximum flow $\left(\mathrm{q}_{\max }\right)$, which is the flow at the critical density $\left(\rho_{\mathrm{c}}\right)$, is

$$
\begin{aligned}
\mathrm{q}_{\max } & =\rho_{\mathrm{c}} \cdot \mathrm{v}_{\mathrm{l}, \mathrm{v}} \\
& =\mathrm{v}_{\mathrm{l}, \mathrm{v}} \cdot \frac{\mathrm{v}_{\text {hole }} \cdot \rho_{\mathrm{jam}}}{\left(\mathrm{v}_{\text {hole }}+\mathrm{v}_{\mathrm{l}, \mathrm{v}}\right)}
\end{aligned}
$$

\subsection{Consequences for the link geometry}

In order to function properly, a link modeled with holes needs to have certain geometrical properties. The problem is that large scale assignment networks typically give the maximum (out)flow as each link's most important attribute. However, clearly, the maximum flow on a link can not be larger than the tip of the fundamental diagram triangle. So the maximum density $\left(\rho_{\text {jam }}\right)$ on the link has to be large enough to move the tip far enough up to fulfill this condition, i.e. qmax $_{\max }$ Eq. 5 is at least as large as the link's (out)flow capacity $\mathrm{q}_{1}$, given by the assignment network:

$$
\mathrm{q}_{\mathrm{l}} \leq \mathrm{q}_{\max }=\mathrm{v}_{\mathrm{l}, \max } \cdot \frac{\mathrm{v}_{\text {hole }} \cdot \rho_{\mathrm{jam}}}{\left(\mathrm{v}_{\text {hole }}+\mathrm{v}_{\mathrm{l}, \mathrm{v}}\right)}
$$

Out of these variables, $\rho_{\mathrm{jam}}$ is the only one that is somewhat flexible, since it can be increased by assuming a larger number of lanes, and the number of lanes is rarely a reliable quantity in assignment networks. In consequence,

$$
\begin{aligned}
\rho_{\text {jam }} & \geq \frac{\mathrm{q}_{\text {max }} \cdot\left(\mathrm{v}_{\text {hole }}+\mathrm{v}_{\mathrm{l}, \mathrm{v}}\right)}{\mathrm{v}_{\mathrm{l}, \mathrm{v}} \cdot \mathrm{v}_{\text {hole }}} \\
\mathrm{N}_{\mathrm{PCU}} & \geq \frac{\mathrm{q}_{\max } \cdot \ell_{\mathrm{l}} \cdot\left(\mathrm{v}_{\text {hole }}+\mathrm{v}_{\mathrm{l}, \mathrm{v}}\right)}{\mathrm{v}_{\mathrm{l}, \mathrm{v}} \cdot \mathrm{v}_{\text {hole }}} \\
& =\mathrm{q}_{\max } \cdot \ell_{\mathrm{l}} \cdot\left(\frac{1}{\mathrm{v}_{\mathrm{l}, \mathrm{v}}}+\frac{1}{\mathrm{v}_{\text {hole }}}\right)
\end{aligned}
$$

where $\mathrm{N}_{\mathrm{PCU}}$ is the number of PCU units that can be placed on the link. In consequence, in the simulation the condition 7 is checked for each link, and if the condition is not fulfilled, this link's storage capacity is increased accordingly $4^{4}$

\footnotetext{
${ }^{4}$ As stated early, the assumption is that a link that is assumed to have a certain flow capacity in the assignment network needs to be physically able to process this flow; if this is not the case, the input data must be erroneous and thus be corrected in a plausible way. Maintaining the flow and increasing the storage seems the best way to do this in an assignment context.
} 

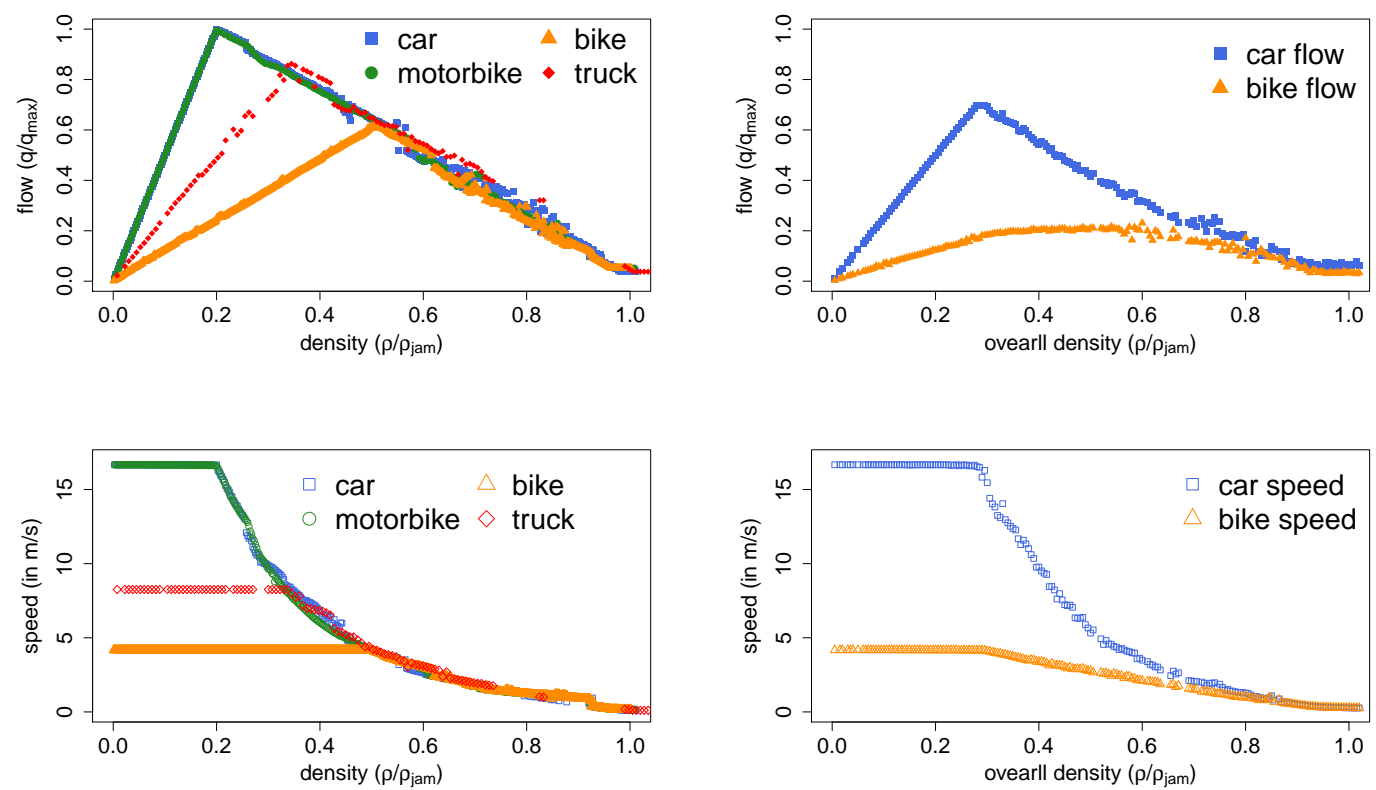

(a) Simulations of single vehicle classes (car, truck, motorbike and bike).

(b) Simulation of equal modal split (in PCU) of car and bike.

Figure 4. FDs for queue model with holes. Adapted after Agarwal and Lämmel (2015).

\subsection{Fundamental diagrams (FDs)}

To understand the traffic flow models and primary relationship between the fundamental variables (q, $\rho$ and $\mathrm{v}$ ), the FDs play an important role. The FDs for queue model without holes are presented in Sec. 2.1. In this section, FDs for queue model with holes are presented for two different link dynamics i.e. FIFO and passing queue.

\subsubsection{Homogeneous traffic (FIFO queue)}

Initially, the FDs for the homogeneous traffic (single vehicle class) simulations, namely car, truck, motorbike, and bike are plotted (see Fig. 4(a)p. For each vehicle class, a different simulation is set up. The resulting FDs are shown in Fig. 4(a).

All vehicle classes show triangular FDs. Car and motorbike have different PCU but the same speed. This results in similar FDs for car and motorbike (points are overlapped on top of each other). Similar overlapping FDs are also obtained for queue model without holes (Agarwal et al. 2015). For truck and bike, a) the maximum flow is achieved at higher density than car and motorbike and b) the maximum flow is lower than maximum flow of car and motorbike. This happens due to their slower speeds. This is also observed while using queue model without holes (see Fig. 2), in which, a decrease in the speed result in the lower maximum flow.

The maximum flow for different vehicles can be estimated using the Eq. 5, i.e. for outflow capacity of $1600 \mathrm{PCU} / \mathrm{h}$, jam density $133.33 \mathrm{PCU} / \mathrm{km}$ and hole speed of $15 \mathrm{~km} / \mathrm{h}$, the normalized maximum flow will be given by $\mathrm{q}_{\max } / 1600=\mathrm{v}_{\mathrm{l,v}}$. $15 \cdot 133.33 /\left(15+\mathrm{v}_{\mathrm{l}, \mathrm{v}}\right) / 1600=1.25 \cdot \mathrm{v}_{\mathrm{l}, \mathrm{v}} /\left(15+\mathrm{v}_{\mathrm{l}, \mathrm{v}}\right)$. This yields to normalized maximum flow of 1.0, 1.0, 0.833, 0.625 for car, motorbike, truck and bike respectively; these values match with the flow values in the Fig. 4(a). 


\subsubsection{Heterogeneous traffic (Passing queue)}

To simulate the heterogeneous traffic conditions, the queue model with holes is also applied to the passing link dynamics. Car and bike vehicle classes are simulated in the equal modal split (in PCU units) and the resulting FDs are shown in Fig. 4(b). The maximum flow of the car vehicle class is higher than the bike vehicle class in a combined simulation because i) car is faster than bike and ii) car can overtake the bike. Further, due to the slower speed of the bike, the maximum flow of the bike is achieved at a density higher than that of for the car.

The critical density at which the car flow is maximum can be estimated by equating the car flows in free flow and congested regimes, i.e., $\mathrm{v}_{\mathrm{l}, \mathrm{v}} \cdot \frac{\rho}{2}=\left(\rho_{\mathrm{jam}}-\frac{\rho}{2}-\frac{\rho}{2}\right) \cdot \mathrm{v}_{\text {hole }}$, where $\rho$ is total density $\left.\right|^{5}$ This will return $\frac{\rho}{\rho_{\text {jam }}}=0.33$ which is approximately same as shown in Fig. 4(b)

\section{Sensitivity}

In this section, the robustness of the proposed queue model is tested. Sec. 5.1 shows the FDs for the same triangular network set up but with the increased storage capacity without changing the link flow capacity. Sec. 5.2 demonstrate the sensitivity towards modal split between car and bike with reference to the flow-density contours. Similarly, average bike passing rate contours are also presented in the Sec. 5.3. These contour plots are also generated using the same experimental set up as described in Sec. 3. In this experiment, car and bike vehicle classes are used for different modal split combinations. For each modal split combination, a separate simulation set up is created.

\subsection{Storage capacity}

In the real world, a motorway is with two lanes and uninterrupted flow whereas an arterial is 2 lane with traffic signal at the end. The ratio of the flow capacity to the storage capacity would be less for the arterials than for the motorway. To incorporate this, we test the effect of more lanes $(=$ a higher storage capacity while maintaining the same outflow) on the queue model with holes is shown in Fig. 5. In the current travel demand simulation framework, lane changing behavior is not modeled, however, the passing (Agarwal et al. 2015) and seepage (Agarwal and Lämmel 2015) link dynamics are included in it.

\subsubsection{Homogeneous traffic}

In Fig. 5(a) also, the axes are normalized to compare it with Fig. 4(a). It can be observed that, due to increased density, the maximum flow is capped by the link (out)flow capacity. That is, the flow in Eq. 3 can be re-written as:

$$
\mathrm{q}= \begin{cases}\mathrm{v}_{\mathrm{l}, \mathrm{v}} \cdot \rho, & \text { if } \rho \leq \rho_{\mathrm{c}_{1}}, \quad \text { (free flow) } \\ \mathrm{q}_{1} & \text { if } \rho_{\mathrm{c}_{1}}<\rho \leq \rho_{\mathrm{C}_{2}}, \text { (constant flow) } \\ \mathrm{v}_{\text {hole }} \cdot\left(\rho_{\mathrm{jam}}-\rho\right), & \text { if } \rho_{\mathrm{c}_{2}}<\rho \leq \rho_{\mathrm{jam}} \text { (congested) }\end{cases}
$$

\footnotetext{
${ }^{5}$ One should note that in this case, equal modal split (in PCU) of car and bike modes are simulated.
} 

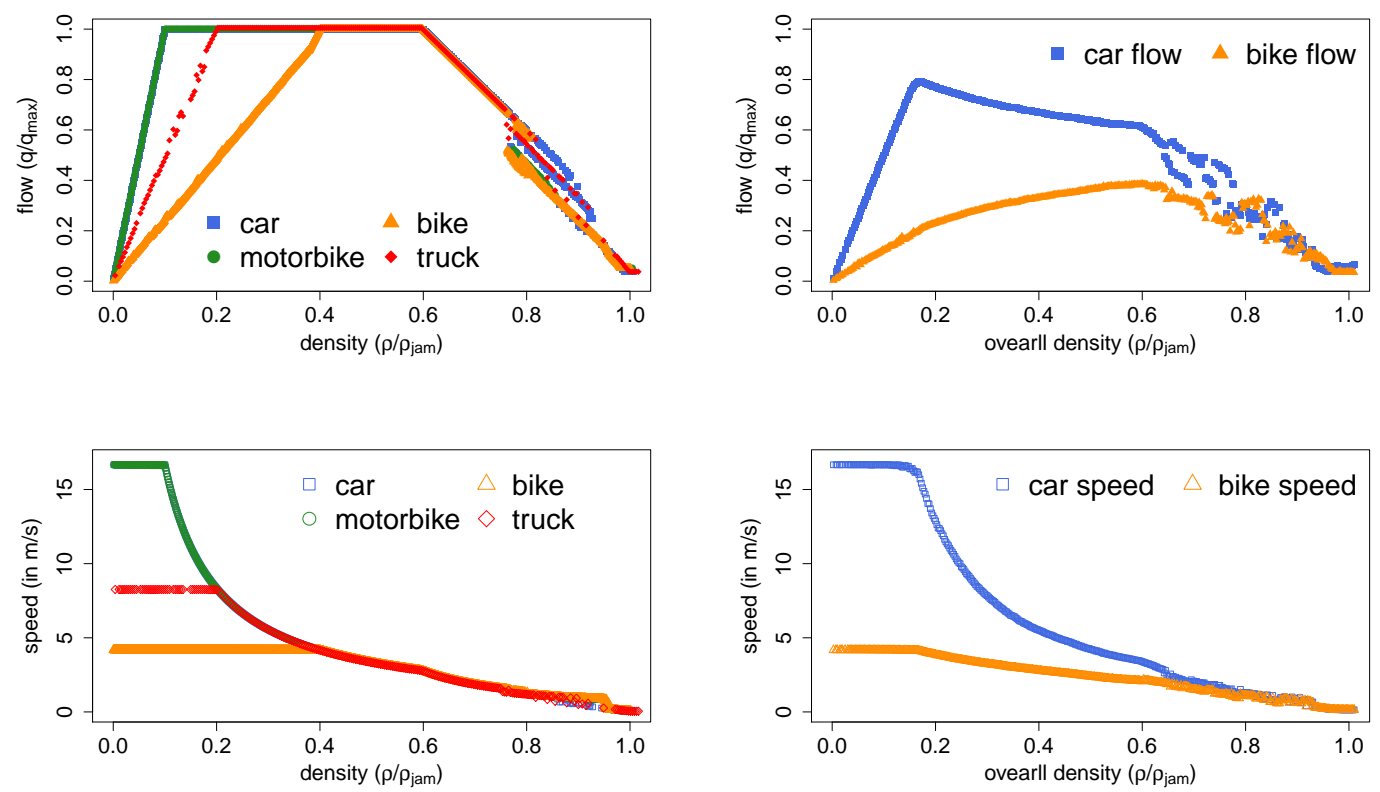

(a) Simulations of single vehicle classes (car, truck, motorbike and bike).

(b) Simulation of equal modal split (in PCU) of car and bike.

Figure 5. FDs for queue model with holes and storage capacity equivalent to 2 lanes.

where $\rho_{\mathrm{C}_{1}}$ and $\rho_{\mathrm{C}_{2}}$ can be estimated as

$$
\rho_{\mathrm{c}_{1}}=\frac{\mathrm{q}_{\mathrm{l}}}{\mathrm{v}_{\mathrm{l}, \mathrm{v}}} \text { and } \rho_{\mathrm{c}_{2}}=\rho_{\mathrm{jam}}-\frac{\mathrm{q}_{\mathrm{l}}}{\mathrm{v}_{\text {hole }}} .
$$

From Eq. 9, normalized value of $\rho_{\mathrm{C}_{2}}$ comes out to 0.2 and it is independent of the vehicle class i.e., the density at which the flow starts decreasing is same for all vehicle classes (see Fig. 5(a) . The critical densities at which the flow become constant are 0.1, 0.1, 0.2, 0.4 for car, motorbike, truck and bicycle respectively. These values are same as shown in Fig. 5(a). The horizontal sections in the FDs for truck and bike are shorter in the present example due to their lower maximum speeds 6

\subsubsection{Heterogeneous traffic}

Fig. 5(b) shows the FDs for car and bike simulation with storage capacity equivalent to two lanes. The maximum flow for car and bike mode is higher than the corresponding flows in the one lane FDs. Moreover, the overall flow (car flow and bike flow) is capped by the link capacity i.e. $1600 \mathrm{PCU} / \mathrm{h}$ (similar to the car and motorbike FDs in Fig. $5(\mathrm{a})$ ). However, the flow density profile for car does not have a horizontal section after reaching the maximum flow rather it starts decreasing at a slow pace due to increase in the flow of the bike vehicle class. Similar to Sec. 4.5.2, the critical densities at which the two transitions of car flow take place, can be estimated using the Eq. 8. That is, (a) the density $\left(\rho_{\mathrm{c}_{1}}\right)$ at which the car flow reaches its maximum flow $\mathrm{v}_{\mathrm{l}, \mathrm{v}} \cdot \frac{\rho_{\mathrm{c}_{1}}}{2}=\mathrm{q}_{\mathrm{l}} \Rightarrow \frac{\rho_{\mathrm{c}_{1}}}{\rho_{\mathrm{jam}}}=0.2$ and, (b) the density $\left(\rho_{\mathrm{C}_{2}}\right)$ at which the car flow starts decreasing rapidly $\mathrm{q}_{\mathrm{l}}=\mathrm{v}_{\text {hole }}$. $\left(\rho_{\mathrm{jam}}-\frac{\rho}{2}-\frac{\rho}{2}\right) \Rightarrow \frac{\rho_{\mathrm{c} 2}}{\rho_{\mathrm{jam}}}=0.6$.

\footnotetext{
${ }^{6}$ One important consequence of the higher storage capacities is that at higher densities, queue model with holes
} displays higher fluctuations. 


\subsection{Flow density contours}

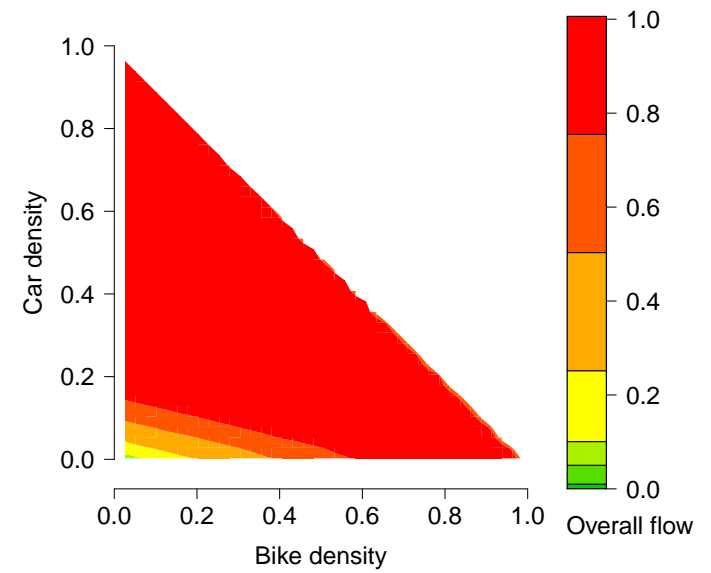

(a) Queue model without holes.

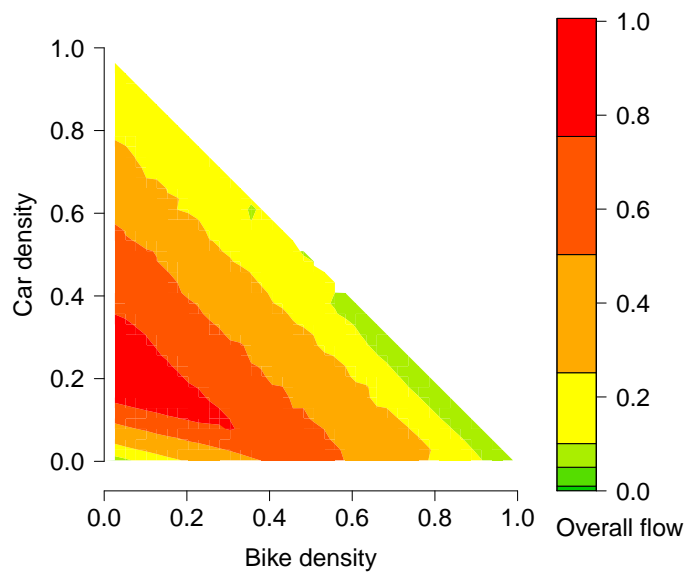

(b) Queue model with holes.

Figure 6. Flow density contours for car bike simulation using passing link dynamics.

Fig. 6 shows the flow density contours for the queue models with and without holes. In Fig. 6(a), flow density contours for queue model without holes are shown. Clearly, due to the nearly vertical slope at higher densities, one can observe that at diagonal values (higher density points), the jammed regime is not realistic and the flow values are equivalent to the link outflow capacity (see Fig. 3 and Fig. 2). The effect of the introduction of the holes in the queue model is clearly visible in Fig. 6(b), The jammed density is clearer than in the queue model without holes. A similar contour plot is presented by Zhang and Jin (2002) in which the authors extend the LWR model analytically for the mixed traffic conditions. Additionally, it can be observed that at the lower densities, i.e. in the free flow regime, similar to the FDs in Fig. 3, both contour plots look equivalent (car density $<=0.1$ and bike density $<=0.4$ ).

\subsection{Average bike passing rate contours}

Fig. 7(a) and Fig. 7(b) show the average bike passing rate contours for the queue model with and without holes respectively. The average bike passing rate is defined as the average number of bikes passed by one car on a one $\mathrm{km}$ road segment. As expected, from the figures, it can be observed that the average bike passing rate is lower in the queue model with holes than in the queue model without holes. Again due to the nearly vertical slope at the higher density for the queue model without holes, the average bike passing rate at the higher bike density is unrealistically high. On the contrary, in the queue model with holes, the average bike passing rate increases with an increase in the bike density and decreases afterwards.

\section{Spatio-temporal plots}

In order to understand and differentiate the queue model with and without holes, space time trajectories are presented in this section. As described before in Sec. 2.1, the queue model controls the agents only during link entry/exit to make it computationally faster. 


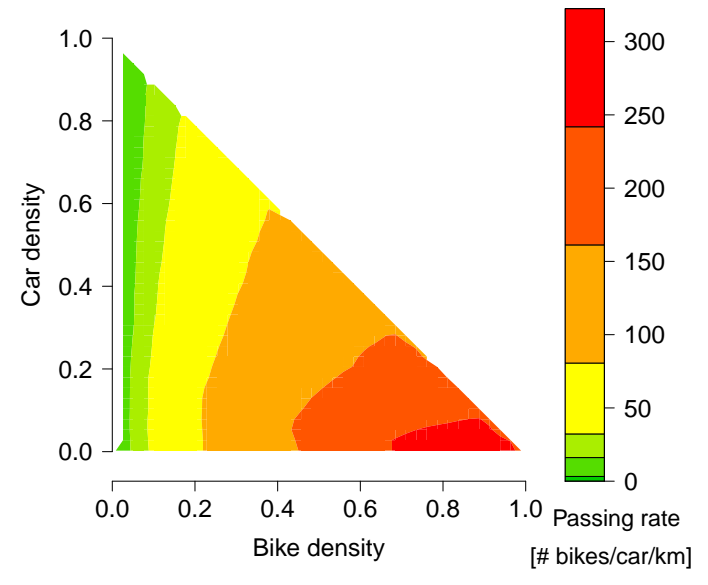

(a) Queue model without holes.

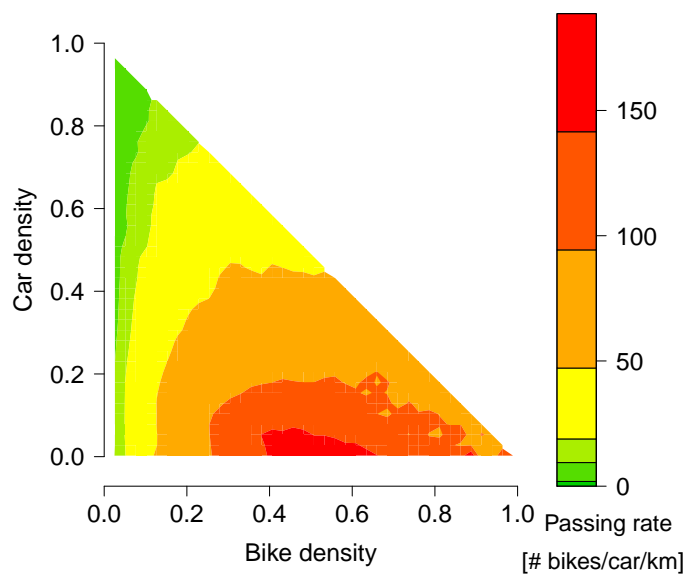

(b) Queue model with holes.

Figure 7. Average bike passing rate contours for car bike simulation using passing link dynamics.

Therefore, these trajectories are plotted by interpolating the intermediate points as described in this section. For simplicity, only car mode is simulated on the triangular race track (see Sec. 3) with minor modification i.e. a hypothetical bottleneck is created to stop the outflow on the middle link for 5 min (between $t=2100$ and $t=2400$ ). This is done in order to observe the queuing pattern. Fig. 8 shows the corresponding space-time trajectories. Following steps are used to generate intermediate points on the link at every time step (=1 sec).

(1) It can be safely assumed that the positions of the backward traveling holes are not affected by the positions of vehicles. It means, once a hole is generated, it will keep traveling with constant speed until it reaches upstream end of the link.

(2) In a capacity/congested regimes, a link is filled with vehicles and holes.

(3) Thus, the positions of the vehicles can be determine as follows.

(a) First, the position of the hole $\left(\mathrm{x}_{\mathrm{t}, \text { hole }}\right)$ from the upstream end of the link is computed using the queue logic.

$$
\mathrm{x}_{\mathrm{t}, \text { hole }}=\left(\frac{\mathrm{t}_{\text {remaining, hole }}}{\mathrm{t}_{\text {hole }}}\right) \cdot \ell_{\mathrm{l}}
$$

where $\mathrm{t}_{\text {remaining, hole }}$ is the time remaining for the hole to reach the upstream end of the link and $t_{\text {hole }}$ is the minimum time required for the hole to reach the upstream end of the link (see Eq. 2).

(b) The position of the vehicle $\left(\mathrm{x}_{\mathrm{t}, \mathrm{veh}}\right)$ from the upstream end of the link is also determined using the queue logic.

$$
\mathrm{x}_{\mathrm{t}, \mathrm{veh}}=\left(1-\frac{\mathrm{t}_{\text {remaining,veh }}}{\mathrm{t}_{l, \text { free }}}\right) \cdot \ell_{\mathrm{l}}
$$

where, $\mathrm{t}_{\text {remaining,veh }}$ is the time remaining to reach the downstream end of the link and $t_{\text {free }}$ is the minimum time for the vehicle to reach the downstream end of the link (see Eq. 1).

(c) Now, for each vehicle, identify the holes such that

$$
\mathrm{x}_{\mathrm{t}, \mathrm{hole}} \geq \mathrm{x}_{\mathrm{t}, \mathrm{veh}}
$$




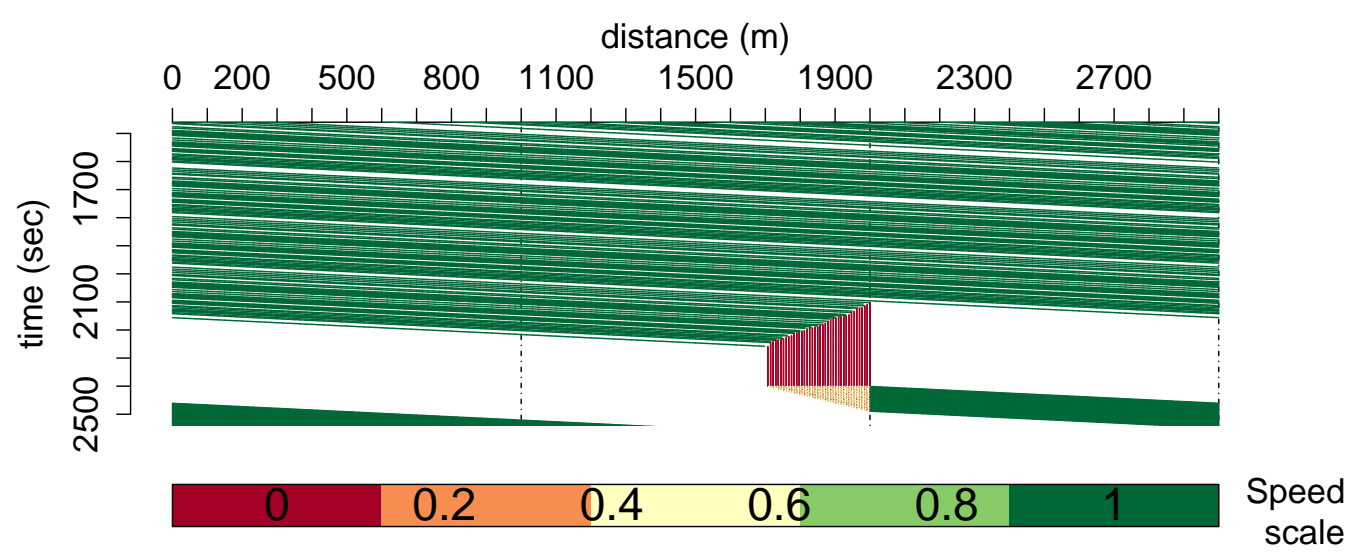

(a) Queue model without holes

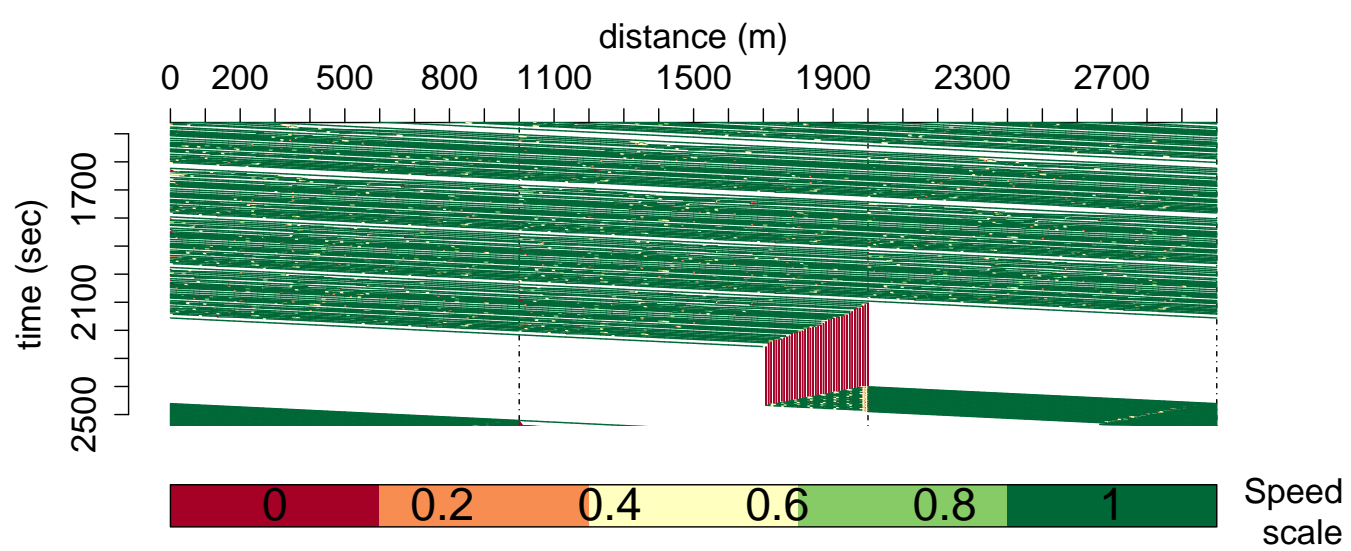

(b) Queue model with holes

Figure 8. Space time trajectories at density $13.33 \mathrm{PCU} / \mathrm{km}$.

and then for each such holes, the vehicle position is recalculated as

$$
\mathrm{x}_{\mathrm{t}, \mathrm{veh}}=\mathrm{x}_{\mathrm{t}, \mathrm{veh}}-\mathrm{PCU}_{\mathrm{hole}} \cdot \text { Size }_{\mathrm{PCU}}
$$

where $\mathrm{PCU}_{\text {hole }}$ and Size $\mathrm{PCU}$ are the PCU equivalent of the respective hole and size of the 1 PCU on the link respectively. Thus, all the vehicles are positioned on the link.

Fig. 8(a) and Fig. 8(b) show the spatio-temporal plots for queue model without holes and with holes respectively $]^{7}$ As soon as the outflow of the middle link come to a halt, the queuing starts. However, as expected, the queuing pattern in both models is different. In the queue model without holes, the position of the leading vehicle is occupied immediately by the following vehicle, consequently, a shock is observed at $t=2400$ in the queue model without holes (see Fig. 8(a) . On the contrary, in the queue model with holes, space is occupied by the following vehicle after some time thus shock is absent at $t=2400$ (see Fig. 8(b) . Subsequently, this results in the higher queue dissipation time. Hence, the proposed approach produces the realistic traffic flow patterns.

\footnotetext{
${ }^{7}$ These trajectories are derived in post-processing, therefore, minor rounding errors can be observed in the trajec-
} tories. 


\section{Computation performance}

In order to check the computational performance, a real-world scenario of Patna, India is taken. This scenario is taken from a previous study by Agarwal et al. (2013) and described here briefly.

\subsection{Set up}

\subsubsection{Link dynamics}

The computational performances of the queue model (with and without holes) with three link dynamics are compared. Three link dynamics are FIFO, passing and seepage. The FIFO and passing link dynamics are explained in Sec. 2.1.1 and Sec. 2.1.2. The seepage link dynamics is explained next.

In mixed traffic streams, due to the smaller size and higher maneuverability, the smaller vehicles (for e.g., bike, motorbike) seep across the gaps between the stationary or almost stationary vehicles, this is called seepage (see, Agarwal and Lämmel 2015, 2016, for methodology and further details). Eventually this means, passing let the faster vehicles overtake the slower vehicles in un-congested regime whereas seepage let the smaller vehicles overtake the larger vehicles in congested regime. This is a common practice in most of the developing nations.

\subsection{2. scenario set up}

The simplified network has 702 nodes and 1934 links. The 1\% sample size has 13122 persons and each person make two trips per day. The scenario has about $2 \%$ car, $37 \%$ bike, $13.88 \%$ motorbike, $17.8 \%$ public transport and $29.2 \%$ walk trips. Car, motorbike and bike are physically simulated on the network and therefore considered as main congested modes for the scenario. Each simulation run is iterated for 200 iterations. In order to compare the performance between the different sample sizes, same experiment is repeated again with the $10 \%$ sample. The synthetic demand is generated by cloning the $1 \%$ population sample with some randomness.

From three link dynamics and two queue models (with and without holes), 6 cases are considered (see X-axis of Fig. 9). Each case is run for 12 different random seeds to check the robustness. In the analysis, two initial runs are omitted from the computation calculation in order to avoid initial scenario start-up related effects.

\subsection{Computational infrastructure}

These simulation runs are processed on a machine which has Super-micro X9DRT type main board, two CPUs with 6 core each (Intel Xeon E5-2630v2) and 64 GB of DDR3 memory clocked at $1600 \mathrm{MHz}$. However, for each run, 4 cores each with $8 \mathrm{~GB}$ is assigned. The experimental runs are performed between 08 February to 23 February 2016.

\subsection{Outcome}

The resulting simulation time for the $1 \%$ sample is shown in Fig. 9(a). Clearly, queue model without holes for FIFO and passing are fastest and take only about 9.8 sec for each iteration. Thus, the computational efficiency is maintained while allowing passing. The introduction of the holes increases simulation time per iteration by less than $1 \mathrm{sec}$, it indicates that the queue model with holes is not resource hungry. This minor increase 


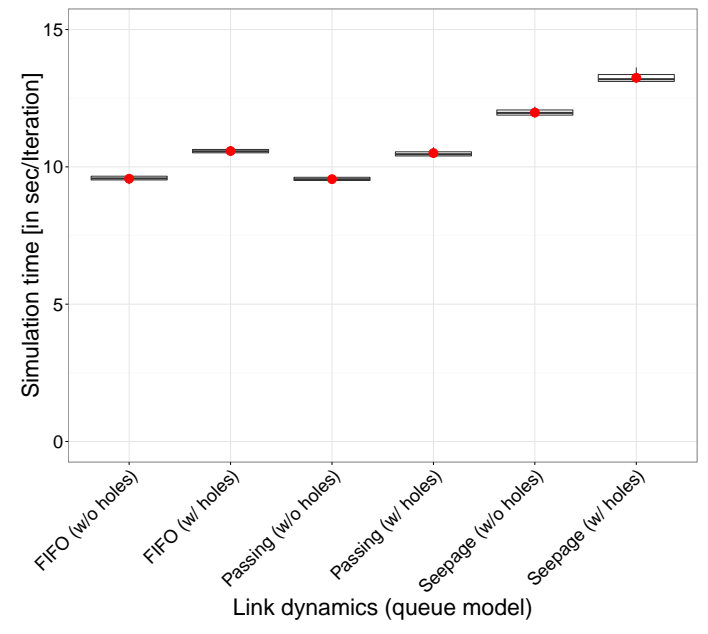

(a) $1 \%$ sample.

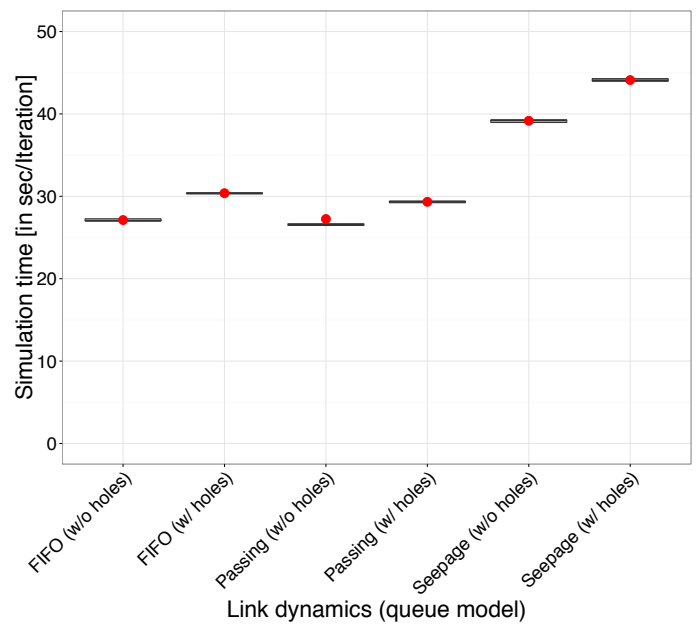

(b) $10 \%$ sample.

Figure 9. Simulation time for 200 runs of Patna scenario (red points show the average values).

in simulation time is originated by an additional data structure to manage the holes. On the contrary, simulation time for seepage link dynamics is increased by more than 2 sec. This is due to the algorithm in seepage where seep mode is searched in the data structure of every link of the network (Agarwal and Lämmel 2015, 2016).

Further, Fig. 9(b) shows the comparison of the simulation time for the $10 \%$ sample size ${ }^{8}$ The simulation time for the $10 \%$ sample sizes shows the similar trend as that of the $1 \%$ sample size for the various traffic and link dynamics of the queue model. Interestingly, one important observation is that the simulation time from $1 \%$ sample size (about $9.8 \mathrm{sec}$ ) to $10 \%$ sample size (about $28 \mathrm{sec}$ ) rises by 2.8 times only.

Thus, to summarize above: a) more than 26,000 trips can be simulated in less than $10 \mathrm{sec}$, it means the proposed approach is suitable for the large-scale scenarios and b) a 10 fold increase in the sample size increases simulation time by a factor of 3 only; this highlights the advantage of using the proposed approach for the different samples $9 \mathrm{pf}$ the large-scale scenarios.

\section{Conclusion and outlook}

Queue models play an important role for the simulation of the large scale scenarios. This study presented a spatial queue model embedded in a multi-agent simulation framework. It controls agents at entry/exit of the link which make it computationally fast. The original queue model was then extended for a more realistic behavior by introducing backward traveling holes for mixed traffic conditions. In this approach, the spaces freed by leaving vehicles are not occupied by the following vehicles immediately which made the queue model closer to reality. The application of the queue model is not limited to an agent-based framework, it can be applied to any other framework/models that displays kinematic waves.

Moreover, first, fundamental diagrams for homogeneous traffic conditions (only one vehicle class) were presented. Afterwards, to test the heterogeneous traffic behavior,

\footnotetext{
${ }^{8} \mathrm{~A}$ few outliers are excluded which show very high simulation time for no reason. This is an artefact due to some unknown external job on the system.

${ }^{9}$ MATSim can produce good results even with $1 \%$ sample size population if schedule-based transit assignment is not used (Nagel 2008 2011).
} 
combination of car and bike was simulated and corresponding fundamental diagrams were presented. The sensitivity of the model was tested with the help of the fundamental diagram for higher storage capacities and, flow density and average bike passing rate contour plots. The contour plots were compared with the queue model without holes. The space-time trajectories were presented and a clear difference in the queuing pattern was observed between queue model with holes and without holes. The computational efficiency of the queue model for various link and traffic dynamics was compared. It was found that due to an additional data structure, the simulation time for the queue model with holes was increased marginally. Interestingly, the simulation time for simulating the $10 \%$ of sample was only about 2.8 times higher than the simulation time for simulating the $1 \%$ of sample size. Thus, the presented queue model is able to simulate heterogeneous traffic conditions more realistically while maintaining the computational performance.

In future, the authors wish to compare the simulation results with that of the results from field surveys. In the proposed approach, a constant speed of backward traveling holes is assumed. However, it can be scenario specific and therefore, it needs to be calibrated from the field studies before applying to the real-world scenarios. The present study compares performance of the link and traffic dynamics of queue model in MATSim framework. However, the comparison of queue model performance (accuracy and performance) with other kinematic wave models is also a future task.

\section{Acknowledgments}

Part of the material was presented in a preliminary form at $11^{\text {th }}$ Traffic Granular Flow (TGF)'15, Nootdorp. The support given by DAAD (German Academic Exchange Service) to Amit Agarwal for his PhD studies at Technische Universität Berlin is greatly acknowledged. The authors also wish to thank H. Schwandt and N. Paschedag at the Department of Mathematics (Technische Universität Berlin), for maintaining our computing clusters.

\section{References}

Agarwal, A., and G. Lämmel. 2015. "Seepage of smaller vehicles under heterogeneous traffic conditions." Procedia Computer Science 52 (C): 890-895.

Agarwal, A., and G. Lämmel. 2016. "Modeling seepage behavior of smaller vehicles in mixed traffic conditions using an agent based simulation." Transp. in Dev. Econ. 2 (2): 1-12.

Agarwal, A., M. Zilske, K.R. Rao, and K. Nagel. 2013. "Person-based dynamic traffic assignment for mixed traffic conditions." In Conference on Agent-Based Modeling in Transportation Planning and Operations, Blacksburg, Virginia, USA. Also VSP WP 12-11, see http://www.vsp.tu-berlin.de/publications

Agarwal, A., M. Zilske, K.R. Rao, and K. Nagel. 2015. "An elegant and computationally efficient approach for heterogeneous traffic modelling using agent based simulation." Procedia Computer Science 52 (C): 962-967.

Balijepalli, N. C., D. Ngoduy, and D. P. Watling. 2014. "The two-regime transmission model for network loading in dynamic traffic assignment problems." Transportmetrica A: Transport Science 10 (7): 563-584.

Balmer, M., B. Raney, and K. Nagel. 2005. "Adjustment of activity timing and duration in an agent-based traffic flow simulation." In Progress in activity-based analysis, edited by H.J.P. Timmermans. 91-114. Oxford, UK: Elsevier.

Balmer, M., M. Rieser, K. Meister, D. Charypar, N. Lefebvre, K. Nagel, and K.W. Axhausen. 2009. "MATSim-T: Architecture and Simulation Times." In Multi-Agent Systems for Traffic and Transportation, edited by A.L.C. Bazzan and F. Klügl. 57-78. IGI Global.

Bliemer, M. C. J. 2007. "Dynamic Queuing and Spillback in Analytical Multiclass Dynamic Net- 
work Loading Model." Transportation Research Record: Journal of the Transportation Research Board 2029: 14-21.

Cetin, N., A. Burri, and K. Nagel. 2003. "A Large-Scale Agent-Based Traffic Microsimulation Based On Queue Model." In Swiss Transport Research Conference (STRC), Monte Verita, Switzerland. See http://www.strc.ch. http://www.strc.ch.

Charypar, D., K.W. Axhausen, and K. Nagel. 2007. "Event-Driven Queue-Based Traffic Flow Microsimulation." Transportation Research Record 2003: 35-40.

Daganzo, C.F. 1994. "The cell transmission model: A dynamic representation of highway traffic consistent with the hydrodynamic theory." Transportation Research B 28B (4): 269-287.

Daganzo, C.F. 1995. "The cell transmission model, part II: Network traffic." Transportation Research B 29B (2): 79-93.

Eissfeldt, N., D. Krajzewicz, K Nagel, and P. Wagner. 2006. "Simulating traffic flow with queues." See http://www.vsp.tu-berlin.de/publications

Flötteröd, G. 2016. "Queueing Representation of Kinematic Waves." In Horni, Nagel, and Axhausen (2016). chap. 50. http://matsim.org/the-book.

Frederix, R., F. Viti, and C.M.J. Tampère. 2010. "A density-based dynamic OD estimation method that reproduces within-day congestion dynamics." In Intelligent Transportation Systems (ITSC), 2010 13th International IEEE Conference on, 694-699.

Gawron, C. 1998. "An Iterative Algorithm to Determine the Dynamic User Equilibrium in a Traffic Simulation Model." International Journal of Modern Physics C 9 (3): 393-407.

Han, Ke, Benedetto Piccoli, and W.Y. Szeto. 2015. "Continuous-time link-based kinematic wave model: formulation, solution existence, and well-posedness." Transportmetrica B: Transport Dynamics 4 (3): 187-222.

Horni, A., K. Nagel, and K. W. Axhausen, eds . 2016. The Multi-Agent Transport Simulation MATSim. Ubiquity, London. http://matsim.org/the-book

Hurdle, V. F., and B. Son. 2001. Shock wave and cumulative arrival and departure models: Partners without conflict. Annual Meeting Preprint 01-2189. Washington, D.C.: Transportation Research Board.

Kim, H., Y. Lim, and K. Lim. 2003. "Two traffic flow models for dynamic network loading." Journal of Eastern Aisa Society for transportation Studies 5.

Lighthill, M. J., and J. B. Whitham. 1955. "On kinematic waves. I: Flow movement in long rivers." Proceedings of the Royal Society A 229: 281-345.

Maciejewski, M. 2010. "Parametric calibration of queue-based traffic flow model in MATSim." In VII Konferencja Naukowo-Techniczna "Systemy Transportowe - Teoria i Praktyka", .

Maerivoet, Sven, and Bart De Moor. 2005. "Transportation Planning and traffic flow models." ArXiv Physics e-prints.

Nagel, K. 2008. Towards simulation-based sketch planning: Some results concerning the Alaskan Way viaduct in Seattle WA. VSP Working Paper 08-22. TU Berlin, Transport Systems Planning and Transport Telematics. See http://www.vsp.tu-berlin.de/publications https://svn.vsp. tu-berlin.de/repos/public-svn/publications/vspwp/2008/08-22.

Nagel, K. 2011. Towards simulation-based sketch planning, part II: Some results concerning a freeway extension in Berlin. VSP Working Paper 11-18. TU Berlin, Transport Systems Planning and Transport Telematics. See http://www.vsp.tu-berlin.de/publications. https: //svn.vsp.tu-berlin.de/repos/public-svn/publications/vspwp/2011/11-18.

Newell, G.F. 1993. "A simplified theory of kinematic waves in highway traffic. I: General theory. II: Queueing at freeway bottlenecks. III: Multi-destination flows." Transportation Research B 27B: 281-313.

Nie, Xiaojian, and H. M. Zhang. 2005. "A comparative study of some macroscopic link models used in dynamic traffic assignment." Networks and Spatial Economics 5 (1): 89-115.

Raney, B., and K. Nagel. 2004. "Iterative Route Planning for Large-Scale Modular Transportation Simulations." Future Generation Computer Systems 20 (7): 1101-1118.

Raney, B., and K. Nagel. 2006. "An improved framework for large-scale multi-agent simulations of travel behaviour." In Towards better performing European Transportation Systems, edited by P. Rietveld, B. Jourquin, and K. Westin. 305-347. London: Routledge.

Richards, P.I. 1956. "Shock waves on the highway." Operations Research 4 (1): 42-51.

Simon, P.M., J. Esser, and K. Nagel. 1999. "Simple queueing model applied to the city of Port- 
land." International Journal of Modern Physics 10 (5): 941-960.

Simon, P.M., and K. Nagel. 1999. Simple queueing model applied to the city of Portland. Annual Meeting Preprint 99-0861. Washington, D.C.: Transportation Research Board.

Smits, Erik-Sander, Michiel Bliemer, and Bart van Arem. 2011. "Dynamic network loading of multiple user-classes with the link transmission model." In 2nd International Conference on Models and Technologies for Intelligent Transportation Systems, No. 057. Leuven, Belgium.

Sumalee, A., R.X. Zhong, T.L. Pan, and W.Y. Szeto. 2011. "Stochastic cell transmission model (SCTM): a stochastic dynamic traffic model for traffic state surveillance and assignment." Transportation Research Part B: Methodological 45 (3): 507 - 533.

Tordeux, Antoine, Michel Roussignol, Jean-Patrick Lebacque, and Sylvain Lassarre. 2014. "A stochastic jump process applied to traffic flow modelling." Transportmetrica A: Transport Science 10 (4): 350-375.

Treiber, Martin, and Arne Kesting. 2013. Traffic flow dynamics. Springer-Verlag Berlin Heidelberg.

United Nations. 2014. World Urbanization prospects : The 2014 revision, highlights. Technical Report ST/ESA/SER.A/352. Department of Economic and Social Affairs, Population Division. van Wageningen-Kessels, Femke, Hans van Lint, Serge Hoogendoorn, and Kees Vuik. 2014. "A new generic multi-class kinematic wave traffic flow model: model development and analysis of its properties." Transportation Research Record: Journal of the Transportation Research Board 2422: 50-60.

Venkatesan, K., A. Gowri, and R. Sivanandan. 2008. "Development of microscopic simulation model for heterogeneous traffic using object oriented approach." Transportmetrica 4 (3): 227247.

Yperman, Isaak. 2007. "The link transmission model for dynamic network loading." Ph.D. thesis. Katholieke Universiteit Leuven.

Zhang, H. M., and W. L. Jin. 2002. "Kinematic wave traffic flow model for mixed traffic." Transportation Research Record: Journal of the Transportation Research Board 1802: 197-204.

Zhang, H. M., and Y. Nie. 2005. "Modeling network flow with and without link interaction: properties and implication." Presented at the 84th annual meeting of the Transportation Research Board.

Zhang, H. M., Yu (Marco) Nie, and Zhen (Sean) Qian. 2013. "Modelling network flow with and without link interactions: the cases of point queue, spatial queue and cell transmission model." Transportmetrica B: Transport Dynamics 1 (1): 33-51.

Zhou, Xuesong, and Jeffrey Taylor. 2014. "DTALite: A queue-based mesoscopic traffic simulator for fast model evaluation and calibration." Cogent Engineering 1 (1): 961345. 


\section{List of Figures}

Figure 1. MATSim evolutionary algorithm and, various traffic and link dynamics.

Figure 2. Flow density curves for car and bike.

Figure 3. Comparison of FDs for only car simulation using queue model with and without holes.

Figure 4. FDs for queue model with holes. Adapted after Agarwal and Lämmel (2015).

a Simulations of single vehicle classes (car, truck, motorbike and bike).

b Simulation of equal modal split (in PCU) of car and bike.

Figure 5. FDs for queue model with holes and storage capacity equivalent to 2 lanes.

a Simulations of single vehicle classes (car, truck, motorbike and bike).

b Simulation of equal modal split (in PCU) of car and bike.

Figure 6. Flow density contours for car bike simulation using passing link dynamics.

a Queue model without holes.

b Queue model with holes.

Figure 7. Average bike passing rate contours for car bike simulation using passing link dynamics.

a Queue model without holes.

b Queue model with holes.

Figure 8. Space time trajectories at density $13.33 \mathrm{PCU} / \mathrm{km}$.

a Queue model without holes.

b Queue model with holes.

Figure 9. Simulation time for 200 runs of Patna scenario (red points show the average values).

a $1 \%$ sample.

b $10 \%$ sample. 